Pacific Journal of Mathematics

NONTANGENTIAL LIMIT THEOREMS FOR NORMAL 


\title{
NON-TANGENTIAL LIMIT THEOREMS FOR NORMAL MAPPINGS
}

\author{
KYONG T. HAHN
}

\begin{abstract}
Let $X$ be a relatively compact complex subspace of a hermitian manifold $N$ with hermitian distance $d_{N}$. Let $\Omega$ be a bounded domain with $C^{1}$-boundary in $\mathbf{C}^{m}$. A holomorphic mapping $f: \Omega \rightarrow N$, $f(\Omega) \subset X$, is called a normal mapping if the family $\{f \circ \psi: \psi: \Delta \rightarrow \Omega$ is holomorphic $\}, \Delta:=\{z \in \mathrm{C}:|z|<1\}$, is a normal family in the sense of $H$. Wu. Let $\left\{p_{n}\right\}$ be a sequence of points in $\Omega$ which tends to a boundary point $\zeta \in \partial \Omega$ such that $\lim _{n \rightarrow \infty} d_{N}\left(f\left(p_{n}\right), l\right)=0$ for some $l \in \bar{X}$. Two sets of sufficient conditions on $\left\{p_{n}\right\}$ are given for a normal mapping $f: \Omega \rightarrow X$ to have the non-tangential limit value $l$, thus extending the results obtained by Bagemihl and Seidel.
\end{abstract}

1. Introduction. In [2], F. Bagemihl and W. Seidel posed the following question: Given a sequence $\left\{z_{n}\right\}$ in the open unit disc $\Delta$ converging to some $\zeta \in \partial \Delta$ and a meromorphic function $f: \Delta \rightarrow P_{1}(\mathrm{C})$ such that $\lim _{n \rightarrow \infty} f\left(z_{n}\right)=c$ for some $c \in P_{1}(\mathbf{C})$, under what conditions on $f$ and $\left\{z_{n}\right\}$ can $f$ have the limit $c$ along some continuum in $\Delta$ which is asymptotic at $\zeta$ ? They answer this question with two interesting sufficient conditions on $f$ and $\left\{z_{n}\right\}$.

In this paper we extend their results to the higher dimensional case. First we shall introduce a few terminologies.

Let $\Omega$ be a bounded domain with $C^{1}$-boundary in $\mathbf{C}^{m}$. Then at each $\zeta \in \partial \Omega$, the tangent space $T_{\zeta}(\partial \Omega)$ and the unit outward normal vector $\nu_{\zeta}$ are well-defined. We denote by $\mathrm{C} T_{\zeta}(\partial \Omega)$ and $\mathrm{C} \nu_{\zeta}$ the complex tangent space and the complex normal space, respectively. The complex tangent space at $\zeta$ is defined as the $(m-1)$ dimensional complex subspace of $T_{\zeta}(\partial \Omega)$ and given by $C T_{\zeta}(\partial \Omega):=\left\{z \in \mathbf{C}^{m}:(z, w)=0\right.$, $\left.\forall w \in \mathbf{C} \nu_{\zeta}\right\},(z, w)=\sum_{j=1}^{m} z_{j} \bar{w}_{j}$.

We say that a subset $S \subset \Omega$ is asymptotic at $\zeta \in \partial \Omega$ if $\bar{S} \cap \partial \Omega=\{\zeta\}$ and non-tangentially asymptotic at $\zeta$ if $S \subset \Gamma_{\alpha}(\zeta)$ for some $\alpha>1$, where

$$
\begin{gathered}
\Gamma_{\alpha}(\zeta):=\left\{z \in \Omega:|z-\zeta|<\alpha \delta_{\zeta}(z)\right\} \\
\delta_{\zeta}(z)=\min \left\{p(z, \partial \Omega), p\left(z, T_{\zeta}(\partial \Omega)\right\}\right.
\end{gathered}
$$


and $p$ denotes the euclidean distance in $\mathbf{C}^{m}$. In particular, a curve $\gamma:(0,1) \rightarrow \Omega$ is non-tangentially asymptotic at $\zeta$ if $\gamma(t) \in \Gamma_{\alpha}(\zeta)$ for some $\alpha>1$ and all $t \in(0,1)$, and $\lim _{t \rightarrow 1-\gamma} \gamma(t)=\zeta$.

Let $N$ be a connected paracompact hermitian manifold with hermitian metric $h_{N}$ which induces the standard topology of $N$. By $d_{N}$ we denote the distance function associated with $h_{N}$.

$\operatorname{By} \operatorname{Hol}(\Omega, N)$ we denote the space of all holomorphic maps $f: \Omega \rightarrow$ $N$. We say that a mapping $f \in \operatorname{Hol}(\Omega, N)$ has an asymptotic limit $l$ at $\zeta \in \partial \Omega$ along the curve $\gamma$ in $\Omega$, write $\lim _{\gamma \ni z \rightarrow \zeta} f(z)=l$, if $\gamma$ is asymptotic at $\zeta$ and $\lim _{t \rightarrow 1-} d_{N}(f(\gamma(t)), l)=0$, a radial limit $l$ at $\zeta$ if $\lim _{\varepsilon \rightarrow 0+} d_{N}\left(f\left(\zeta-\varepsilon v_{\zeta}\right), l\right)=0$, a non-tangential limit $l$ at $\zeta$ if $\lim _{\Gamma_{n}(\zeta) \ni z \rightarrow \zeta} d_{N}(f(z), l)=0$ for every $\alpha>1$ and an admissible limit $l$ at $\zeta$ if $\lim _{A_{n}(\zeta) \ni z \rightarrow \zeta} d_{N}(f(z), l)=0$ for every $\alpha>0$, where

(2) $A_{\alpha}(\zeta):=\left\{z \in \Omega:\left|\left(z-\zeta, \nu_{\zeta}\right)\right|<(1+\alpha) \delta_{\zeta}(z),|z-\zeta|^{2}<\alpha \delta_{\zeta}(z)\right\}$.

Let $M$ be a connected complex manifold of dimension $m$. We assume that $M$ is hyperbolic, i.e., the Kobayashi pseudometric $k_{M}$ is a metric. Denote the infinitesimal Kobayashi metric by $K_{M}$. According to $\mathrm{H}$. Royden [10], the Kobayashi metric $k_{M}$ is the integrated form of $K_{M} . M$ is hyperbolic if and only if for each $p \in M$, there exists a neighborhood $U_{p}$ and a constant $a_{U}>0$ such that

$$
K_{M}(q, \xi) \geq a_{U}|\xi| \quad \text { for }(q, \xi) \in U \times \mathbf{C}^{m} .
$$

Definition. A mapping $f \in \operatorname{Hol}(M, N)$ is called normal if the family $\{f \circ \psi: \psi \in \operatorname{Hol}(\Delta, M)\}, \Delta$ is the unit disc in $\mathbf{C}$, forms a normal family in the sense of $\mathrm{H}$. Wu [11].

We remark that the definition of normality adopted here does not require $M$ to be homogeneous and coincides with that of [7] when $M$ is homogeneous and $N$ is compact [1], [6]. Therefore, it is a slightly more general notion than that of [7].

2. Preliminary properties of normal mappings. Let $X$ be a relatively compact complex subspace of a hermitian manifold $N$. We shall denote by $\operatorname{Hol}(M, X)$ the space of all holomorphic maps $f: M \rightarrow N$ with $f(M) \subset X$.

LEMMA 1. Let $M$ be a hyperbolic manifold and let $X$ be a relatively compact complex subspace of a hermitian manifold $N$ with hermitian metric $h_{N}$. The family $F \subset \operatorname{Hol}(M, X)$ is normal in the sense of $H$. Wu 
if for each compact subset $E \subset M$ there exists a constant $C(E)>0$ such that

$$
Q f(p):=\sup _{|\xi|=1} \frac{h_{N}(f(p), d f(p) \xi)}{K_{M}(p, \xi)} \leq C(E)
$$

for all $p \in E$ and all $f \in F$.

Due to the compactness of $\bar{X}$, the proof of Lemma 1 can be carried out in the same way as that of Lemma 2.7 of [7]. Therefore, we omit the proof.

THEOREM 1. Let $M$ be a hyperbolic manifold (not necessarily homogeneous) and let $X$ be a relatively compact complex subspace of a hermitian manifold $N$. The following statements are equivalent for $f \in \operatorname{Hol}(M, X)$.

(a) $f$ is normal.

(b) There exists a constant $Q>0$ such that

$$
Q f:=\sup \{Q f(p): p \in M\} \leq Q .
$$

(c) There is no $P$-sequence $\left\{p_{n}\right\}$ in $M$ possessed by $f$, i.e., there is no sequence $\left\{q_{n}\right\}$ in $M$ such that $\lim _{n \rightarrow \infty} k_{M}\left(p_{n}, q_{n}\right)=0$ but $\overline{\lim }_{n \rightarrow \infty} d_{N}\left(f\left(p_{n}\right), f\left(q_{n}\right)\right) \geq \varepsilon$ for some $\varepsilon>0$.

Proof. (a) $\Rightarrow(b)$ : Assume that $\{f \circ \psi: \psi \in \operatorname{Hol}(\Delta, M)\}$ is a normal family. By Lemma 1 , for each compact $E \subset \Delta$, there exists a constant $Q=Q(E)>0$ such that

$$
h_{N}\left(f \circ \psi(0),(f \circ \psi)^{\prime}(0)\right) \leq Q
$$

for all $\psi \in \operatorname{Hol}(\Delta, M)$. By the definition of $K_{M}$ at $(p, \xi) \in M \times \mathbf{C}^{m}$, there exists $\psi \in \operatorname{Hol}(\Delta, M)$ such that $\psi(0)=p, \psi^{\prime}(0) a=\xi$ for $a>0$ and $a / 2<K_{M}(p, \xi) \leq a$. Therefore, from (4),

$$
h_{N}(f(p), d f(p) \xi) \leq 2 Q K_{M}(p, \xi)
$$

for all $(p, \xi) \in M \times \mathbf{C}^{m}$. Namely, $Q f \leq 2 Q$.

(b) $\Rightarrow$ (c): If (c) fails to hold, then there exists a sequence $\left\{p_{n}\right\}$ and $\left\{q_{n}\right\}$ in $M$ with $\lim _{n \rightarrow \infty} k_{M}\left(p_{n}, q_{n}\right)=0$ but $\overline{\lim } d_{N}\left(f\left(p_{n}\right), f\left(q_{n}\right)\right)$ $\geq \varepsilon$ for some $\varepsilon>0$. It contradicts (b), because (b) implies that $d_{N}\left(f\left(p_{n}\right), f\left(q_{n}\right)\right) \leq Q k_{M}\left(p_{n}, q_{n}\right)$.

(c) $\Rightarrow$ (a): If (c) holds, then for every $\varepsilon>0$ there exists a $\delta>0$ such that for all $z, w \in \Delta, k_{\Delta}(z, w)<\delta$ implies $d_{N}(f \circ \psi(z), f \circ \psi(w))<\varepsilon$ 
for all $\psi \in \operatorname{Hol}(\Delta, M)$, since otherwise there exists an $\varepsilon>0$ such that for all $n \in \mathbf{N}$ there exist sequences $\left\{z_{n}\right\}$ and $\left\{w_{n}\right\}$ in $\Delta$ with $k_{\Delta}\left(z_{n}, w_{n}\right)<1 / n$ but $d_{N}\left(f \circ \psi\left(z_{n}\right), f \circ \psi\left(w_{n}\right)\right) \geq \varepsilon$ for some $\psi \in$ $\operatorname{Hol}(\Delta, M)$. This means that $\left\{z_{n}\right\}$ is a $P$-sequence for $f \circ \psi$. Since

$$
k_{M}\left(\psi\left(z_{n}\right), \psi\left(w_{n}\right)\right) \leq k_{\Delta}\left(z_{n}, w_{n}\right) \leq 1 / n \rightarrow 0,
$$

$\left\{\psi\left(z_{n}\right)\right\}$ is also a $P$-sequence for $f$ in $M$ which contradicts (c). Therefore, $\{f \circ \psi: \psi \in \operatorname{Hol}(\Delta, M)\}$ is an equicontinuous family and hence normal since $\bar{X}$ is compact. This proves (a).

Theorem 1 is also proved in [6] for compact $N$ and in [3] for $N=$ the Riemann sphere.

\section{Boundary behavior of normal mappings.}

TheOREM 2. Let $X$ and $N$ be given as in Theorem 1 , and let $\Omega$ be a bounded domain with $C^{1}$-boundary in $\mathbf{C}^{m}$. Suppose that $S$ is an arbitrary asymptotic continuum at $\zeta \in \partial \Omega$ such that

$$
\lim _{S \ni z \rightarrow \zeta} \frac{p\left(z, \mathbf{C} \nu_{\zeta}\right)}{r(\nu(z))}=0
$$

where $r(\nu(z))$ denotes the radius of the largest ball in $\Omega \cap \mathbf{C} T_{\nu(z)}$, centered at $\nu(z)$, the orthogonal projection of $z$ to $\mathrm{C} \nu_{\zeta}$ and $\mathrm{C} T_{\nu(z)}$ is the hyperplane through $\nu(z)$ that is parallel to $\mathrm{C} T_{\zeta}(\partial \Omega)$. If $f \in \operatorname{Hol}(\Omega, X)$ is a normal map such that $\lim _{S \ni z \rightarrow \zeta} d_{N}(f(z), l)=0$ for some $l \in \bar{X}$, then $\lim _{\Gamma_{1}(\zeta) \ni z \rightarrow \zeta} d_{N}(f(z), l)=0$ for all $\alpha>1$.

Proof. By the definition of $r(\nu(z)), \Omega \cap C T_{\nu(z)}$ contains the euclidean ball $\left.B(\nu(z), r(\nu(z)))\right|_{C T_{\nu(-)}}$, the restriction to $\mathrm{C} T_{\nu(z)}$.

The distance-decreasing property of the Kobayashi metric implies

$$
k_{\Omega}(z, \nu(z)) \leq \tanh ^{-1} \frac{|z-\nu(z)|}{r(\nu(z))},
$$

and hence, as $S \ni z \rightarrow \zeta, \eta:=\nu(z) \rightarrow \zeta$ along $\nu(S):=\{\nu(z): z \in S\}$ from (7). Since $f$ is normal, by Theorem 1 , there exists a number $Q>0$ such that

$$
d_{N}\left(f(z), f(\nu(z)) \leq Q k_{\Omega}(z, \nu(z)) .\right.
$$

Therefore, $\lim _{\nu(S) \ni \eta \rightarrow \zeta} d_{N}(f(\eta), l)=0$. Let $\Omega_{\zeta}$ be the connected component of $\Omega \cap C \nu_{\zeta}$ with $\zeta \in \partial \Omega_{\zeta}$. Then the restriction $\left.f\right|_{\Omega_{\zeta}}$ is a normal 
map from the plane domain $\Omega_{\zeta}$ into $X$. Therefore, it follows from Theorem 4 of [5] with a slight modification that

$$
\lim _{\tilde{\Gamma}_{n}(\zeta) \ni \eta \rightarrow \zeta} d_{N}(f(\eta), l)=0 \text { for all } \alpha>1 \text {, }
$$

where $\tilde{\Gamma}_{\alpha}(\zeta):=\Gamma_{\alpha}(\zeta) \cap C \nu_{\zeta}$. The rest of the proof can easily be carried over from the proof of Proposition 8.2 of [7] to this case with $X$ replaced by $d_{N}$.

Corollary 1. Let $X$ and $N$ be given as in Theorem 1 and let $\Omega$ be a bounded domain with $C^{2}$-boundary in $\mathbf{C}^{m}$. Let $S$ be an arbitrary asymptotic continuum at $\zeta \in \partial \Omega$ such that

$$
\lim _{S \ni z \rightarrow \zeta} \frac{p^{2}\left(z, \mathbf{C} \nu_{\zeta}\right)}{p\left(z, \mathbf{C} T_{\zeta}\right)}=0 .
$$

If $f \in \operatorname{Hol}(\Omega, X)$ is a normal map such that $\lim _{S \ni z \rightarrow \zeta} d_{N}(f(z), l)=0$ for some $l \in \bar{X}$, then

$$
\lim _{\Gamma_{n}(\zeta) \ni z \rightarrow \zeta} d_{N}(f(z), l)=0 \text { for all } \alpha>1
$$

Proof. Since $\Omega$ is a bounded domain with $C^{2}$-boundary in $\mathbf{C}^{m}$, there exists an $\varepsilon=\varepsilon(\zeta)>0$ such that the euclidean ball $B_{\varepsilon}:=$ $B\left(\zeta-\varepsilon \nu_{\zeta}, \varepsilon\right)$ is contained in $\Omega$ and tangent to $\partial \Omega$ at $\zeta$ from inside. The order of tangency in this case is not worse than along the admissible region $A_{\alpha}$ given in (2). In fact, there exists a constant $C>0$ such that

$$
r(\nu(z)) \geq C|\zeta-\nu(z)|^{1 / 2}
$$

for $z \in S$. See Example 1 of [4]. Therefore,

$$
\left[\frac{C p\left(z, \mathbf{C} \nu_{\zeta}\right)}{r(\nu(z))}\right]^{2} \leq \frac{|z-\nu(z)|^{2}}{|\zeta-\nu(z)|} \leq \frac{p^{2}\left(z, \mathbf{C} \nu_{\zeta}\right)}{p\left(z, \mathbf{C} T_{\zeta}\right)}
$$

Corollary 1 now follows from Theorem 2 or directly from the Proof of Proposition 8.2 of [7] with minor adjustments.

We now prove the following extensions of the results given in [2].

TheOREM 3. Let $X$ and $N$ be given as in Theorem 1. Let $\Omega$ be a bounded homogeneous domain in $\mathbf{C}^{m}$ and let $\left\{p_{n}\right\}$ be a sequence of points in $\Omega$ which tends to a boundary point $\zeta \in \partial \Omega$ where the outward normal $\nu_{\zeta}$ exists, such that

(a) there exists a constant $M>0$ with $k_{\Omega}\left(p_{n}, p_{n+1}\right) \leq M$ for all $n$,

$$
\lim _{n \rightarrow \infty} \frac{p\left(p_{n}, \mathbf{C} \nu_{\zeta}\right)}{r\left(\nu\left(p_{n}\right)\right)}=0
$$


If $f \in \operatorname{Hol}(\Omega, N)$ is a normal map which omits $l \in \bar{X}$ in $\Omega$ but $\lim _{n \rightarrow \infty} d_{N}\left(f\left(p_{n}\right), l\right)=0$ then

$$
\lim _{\Gamma_{r r}(\zeta) \ni z \rightarrow \zeta} d_{N}(f(z), l)=0 \text { for all } \alpha>1 .
$$

Proof. Let $\varphi_{n} \in \operatorname{Aut}(\Omega)$ be such that $\varphi_{n}\left(p_{0}\right)=p_{n}$ for some fixed point $p_{0} \in \Omega$. Then the family $\left\{g_{n}\right\}, g_{n}=f \circ \varphi_{n}$, omits $l$ for all $n$ and forms a normal family, since $f$ is normal.

For $R>M$, let $B_{k}\left(p_{0}, R\right):=\left\{p \in \Omega: k_{\Omega}\left(p_{0}, p\right)<R\right\}$. Since $\Omega$ is homogeneous, $k_{\Omega}$ is complete and, hence $\bar{B}_{k}\left(p_{0}, R\right)$ is a compact subset of $\Omega$. So, $\left\{g_{n}\right\}$ has a subsequence $\left\{g_{m}\right\}$ which converges uniformly on $\bar{B}_{k}$ to $g \in \operatorname{Hol}(\Omega, N)$. Since each $g_{m}$ omits $l$ on $B_{k}$, by the Hurwitz theorem [8], either $g(z) \neq l$ or $g(z) \equiv l$ on $B_{k}\left(p_{0}, R\right)$. But since $d_{N}\left(g_{m}\left(p_{0}\right), l\right)=d_{N}\left(f\left(p_{m}\right), l\right) \rightarrow 0, g(z) \equiv l$ for all $z \in B_{k}\left(p_{0}, R\right)$. This implies that $f(z)=l$ for all $z \in B_{k}\left(p_{m}, R\right)$ and all $m$, i.e., $f(z) \equiv l$ on $\bigcup_{m=1}^{\infty} B_{k}\left(p_{m}, R\right)$. Since

$$
k_{\Omega}\left(p_{m}, \nu\left(p_{m}\right)\right) \leq \tanh ^{-1} \frac{\left|p_{m}-\nu\left(p_{m}\right)\right|}{r\left(\nu\left(p_{m}\right)\right)} \rightarrow 0
$$

as $n \rightarrow \infty$, there exists $m_{0}$ such that for all $m \geq m_{0} k_{\Omega}\left(p_{m}, \nu\left(p_{m}\right)\right)<R$ which implies $\nu\left(p_{m}\right) \in B_{k}\left(p_{m}, R\right)$ for all $m \geq m_{0}$. Let $S:=C \nu_{\zeta} \cap$ $\bigcup_{m \geq m_{0}} B_{k}\left(p_{m}, R\right)$.

Then condition (6a) in Theorem 2 is trivially satisfied and also $\lim _{S \ni z \rightarrow \zeta} d_{N}(f(z), l)=0$. Therefore, we have

$$
\lim _{\Gamma_{r}(\zeta) \ni z \rightarrow \zeta} d_{N}(f(z), l)=0
$$

for all $\alpha>1$ by Theorem 2 .

Theorem 4. Let $X$ and $N$ be given as in Theorem 1. Let $\left\{p_{n}\right\}$ be a sequence of points in a bounded domain $\Omega \subset \mathbf{C}^{m}$ which tends to a boundary point $\zeta \in \partial \Omega$ where the unit outward normal $\nu_{\zeta}$ exists such that

$$
\begin{gathered}
\lim _{n \rightarrow \infty} k_{\Omega}\left(p_{n}, p_{n+1}\right)=0, \\
\lim _{n \rightarrow \infty} \frac{p\left(p_{n}, \mathbf{C} \nu_{\zeta}\right)}{r\left(\nu\left(p_{n}\right)\right)}=0 .
\end{gathered}
$$

If $f \in \operatorname{Hol}(\Omega, X)$ is a normal map such that $\lim _{n \rightarrow \infty} d_{N}\left(f\left(p_{n}\right), l\right)=0$ for some $l \in \bar{X}$, then $\lim _{\Gamma_{r}(\zeta) \ni z \rightarrow \zeta} d_{N}(f(z), l)=0$ for all $\alpha>1$. 
Proof. Let $\left\{q_{n}\right\}, q_{n}=\nu\left(p_{n}\right)$, be the orthogonal projection of $\left\{p_{n}\right\}$ to $\mathrm{C} \nu_{\zeta}$. Then

$$
k_{\Omega}\left(q_{n}, q_{n+1}\right) \leq k_{\Omega}\left(p_{n}, p_{n+1}\right)
$$

so that $k_{\Omega}\left(q_{n}, q_{n+1}\right) \rightarrow 0$ as $n \rightarrow \infty$. Let $\gamma$ be a curve in $\Omega \cap C \nu_{\zeta}$ joining $q_{n}$ and $q_{n+1}$ by shortest curves. Since $k_{\Omega}$ is an inner metric, such curves exist for sufficiently large $n$. Since $f$ is normal, by Theorem 1 , there exists $Q>0$ such that

$$
d_{N}\left(f\left(p_{n}\right), f\left(q_{n}\right)\right) \leq Q k_{\Omega}\left(p_{n}, q_{n}\right) .
$$

Therefore, condition (b) together with (7) implies

$$
\lim _{n \rightarrow \infty} d_{N}\left(f\left(p_{n}\right), f\left(q_{n}\right)\right)=0,
$$

and hence,

$$
\lim _{n \rightarrow \infty} d_{N}\left(f\left(q_{n}\right), l\right)=0
$$

by the triangle inequality. We wish to show:

$$
\lim _{\gamma \ni z \rightarrow \zeta} d_{N}(f(z), l)=0 .
$$

Suppose there is a sequence $\left\{q_{n}^{\prime}\right\}$ on $\gamma$ converging to $\zeta$ for which $f$ fails to have the limit $l$. By the compactness of $\bar{X}$ there must be a subsequence $\left\{q_{m}^{\prime}\right\}$ such that

$$
\lim _{m \rightarrow \infty} d_{N}\left(f\left(q_{m}^{\prime}\right), l^{\prime}\right)=0
$$

for some $l^{\prime} \in \bar{X}, l^{\prime} \neq l$. We may assume that $q_{m}^{\prime}$ are all distinct from the points $q_{m}$. For each $m$, there exists an index $n_{m}$ such that $q_{m}^{\prime}$ lies on the geodesic segment of $\gamma$ that joins $q_{n_{m}}$ and $q_{n_{m}+1}$. By (10),

$$
k_{\Omega}\left(q_{n_{m}}, q_{m}^{\prime}\right) \leq k_{\Omega}\left(q_{n_{m}} q_{n_{m}+1} \rightarrow 0\right.
$$

as $m \rightarrow \infty$. Since $f$ is normal, for some $Q>0$ we have

$$
d_{N}\left(f\left(q_{n_{m}}\right), f\left(q_{m}^{\prime}\right)\right) \leq Q k_{\Omega}\left(q_{n_{m}}, q_{m}^{\prime}\right) \rightarrow 0
$$

as $m \rightarrow \infty$. From this and (12) we conclude $\lim _{m \rightarrow \infty} d_{N}\left(f\left(q_{m}^{\prime}\right), l\right)=0$, contradicting (14). Therefore we have (13). Since condition (6a) of Theorem 2 holds trivially in this case, Theorem 4 follows from Theorem 2.

We remark that if the domain $\Omega$ in Theorems 3 and 4 is assumed to have $C^{2}$-boundary, then both theorems hold when condition (b) is 
replaced by

$$
\lim _{n \rightarrow \infty} \frac{p^{2}\left(p_{n}, \mathbf{C} \nu_{\zeta}\right)}{p\left(p_{n}, \mathbf{C} T_{\zeta}\right)}=0
$$

in both cases.

Introducing the notion of hypoadmissible limit, J. Cima and S. Krantz have proved the Lindelöf Principle for normal meromorphic functions on domains in $\mathbf{C}^{n}$ with $C^{2}$-boundary in [3]. The author wishes to thank the referee for pointing this out to him.

\section{REFERENCES}

[1] G. Aladro, Some consequences of the boundary behavior of Caratheodory and Kobayashi metrics and applications to normal holomorphic functions, Pennsylvania State University $\mathrm{Ph}$. D. Thesis, 1985.

[2] F. Bagemihl and W. Seidel, Sequential and continuous limits of meromorphic functions, Ann. Acad. Sci. Fenn., Ser. A1, 280 (1960).

[3] J. Cima and S. Krantz, The Lindelöf principle and normal functions of several complex variables, Duke Math. J., 50 (1983), 303-328.

[4] E. Cirka, The theorems of Lindelöf and Fatou in $\mathbb{C}^{n}$, Math. Sb., 92 (134) (1973), 622-644; Math. U.S.S.R. Sb., 21 (1973), 619-639.

[5] K. Funahashi, Normal holomorphic mappings and classical theorems of function theory, Nagoya Math. J., 94 (1984), 89-104.

[6] G. Gigante, Montel's theorem and P-sequences, preprint.

[7] K. T. Hahn, Asymptotic behavior of normal mappings of several complex variables, Canad. J. Math., 36 (1984), 718-746.

[8] P. J. Kiernan and S. Kobayashi, Holomorphic mappings into projective space with lacunary hyperplanes, Nagoya Math. J., 50 (1973), 199-216.

[9] S. Kobayashi, Hyperbolic manifolds and holomorphic mappings, Pure and Appl. Math., 2, Dekker, New York, 1970.

[10] H. L. Royden, Remarks on the Kobayashi Metric, Lecture Notes in Math., 185 (1971), Springer, 125-137.

[11] H. Wu, Normal families of holomorphic mappings, Acta Math., 119 (1967), 193-233.

Received April 24, 1987. Research partially supported by the 1986/1987 Fulbright Research Grant at Universität Osnabrück-Abteilung Vechta.

The Pennsylvania State University UNIVERSITY PARK, PA 16802 


\section{PACIFIC JOURNAL OF MATHEMATICS}

\section{EDITORS}

\author{
V. S. VARADARAJAN \\ (Managing Editor) \\ University of California \\ Los Angeles, CA 90024 \\ HeRBERT ClEMENS \\ University of Utah \\ Salt Lake City, UT 84112 \\ R. FINN \\ Stanford University \\ Stanford, CA 94305
}

\section{HERMANN FLASCHKA \\ University of Arizona \\ Tucson, AZ 85721}

VAUghan F. R. Jones

University of California

Berkeley, CA 94720

ROBION KIRBY

University of California

Berkeley, CA 94720

\author{
C. C. MOORE \\ University of California \\ Berkeley, CA 94720
}

HAROLD STARK

University of California, San Diego

La Jolla, CA 92093

\section{ASSOCIATE EDITORS}
R. ARENS
E. F. BECKENBACH
B. H. NEUMANN
F. WOLF
K. YOSHIDA
(1906-1982)

\section{SUPPORTING INSTITUTIONS}

\begin{abstract}
UNIVERSITY OF ARIZONA
UNIVERSITY OF BRITISH COLUMBIA

UNIVERSITY OF CALIFORNIA

MONTANA STATE UNIVERSITY

UNIVERSITY OF NEVADA, RENO

NEW MEXICO STATE UNIVERSITY

OREGON STATE UNIVERSITY
\end{abstract}
CALIFORNIA INSTITUTE OF TECHNOLOGY

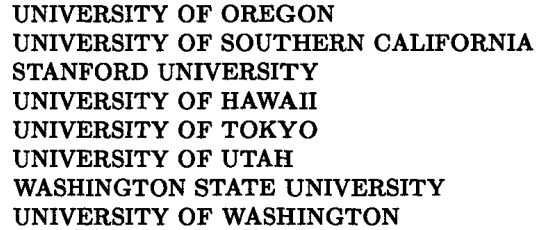

The Supporting Institutions listed above contribute to the cost of publication of this Journal, but they are not owners or publishers and have no responsibility for its content or policies.

Mathematical papers intended for publication in the Pacific Journal of Mathematics should be in typed form or offset-reproduced (not dittoed), double spaced with large margins. Please do not use built up fractions in the text of the manuscript. However, you may use them in the displayed equations. Underline Greek letters in red, German in green, and script in blue. The first paragraph must be capable of being used separately as a synopsis of the entire paper. In particular it should contain no bibliographic references. Please propose a heading for the odd numbered pages of less than 35 characters. Manuscripts, in triplicate, may be sent to any one of the editors. Please classify according to the scheme of Math. Reviews, Index to Vol. 39. Supply name and address of author to whom proofs should be sent. All other communications should be addressed to the managing editor, or Elaine Barth, University of California, Los Angeles, California 90024.

There are page-charges associated with articles appearing in the Pacific Journal of Mathematics. These charges are expected to be paid by the author's University, Government Agency or Company. If the author or authors do not have access to such Institutional support these charges are waived. Single authors will receive $\mathbf{5 0}$ free reprints; joint authors will receive a total of 100 free reprints. Additional copies may be obtained at cost in multiples of 50 .

The Pacific Journal of Mathematics is issued monthly as of January 1966. Regular subscription rate: $\$ 190.00$ a year (5 Vols., 10 issues). Special rate: $\$ 95.00$ a year to individual members of supporting institutions.

Subscriptions, orders for numbers issued in the last three calendar years, and changes of address should be sent to Pacific Journal of Mathematics, P.O. Box 969, Carmel Valley, CA 93924, U.S.A. Old back numbers obtainable from Kraus Periodicals Co., Route 100, Millwood, NY 10546.

The Pacific Journal of Mathematics at P.O. Box 969, Carmel Valley, CA 93924 (ISSN 0030-8730) publishes 5 volumes per year. Application to mail at Second-class postage rates is pending at Carmel Valley, California, and additional mailing offices. Postmaster: send address changes to Pacific Journal of Mathematics, P.O. Box 969, Carmel Valley, CA 93924.

PUBLISHED BY PACIFIC JOURNAL OF MATHEMATICS, A NON-PROFIT CORPORATION Copyright (C) 1988 by Pacific Journal of Mathematics 


\section{Pacific Journal of Mathematics}

Vol. 135, No. $1 \quad$ September, 1988

Margaret M. Bayer, Barycentric subdivisions $\ldots \ldots \ldots \ldots \ldots \ldots \ldots \ldots \ldots$

Eung Chun Cho, $s$-Smith equivalent representations of dihedral groups . . . . 17

Avner Friedman and Jindrich Necas, Systems of nonlinear wave equations

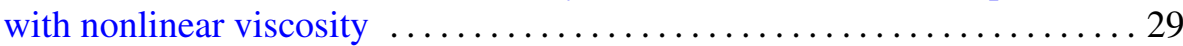

Kyong Taik Hahn, Nontangential limit theorems for normal mappings . . . . 557

Eloise A. Hamann, Evan Green Houston, Jr. and Jon Lee Johnson,

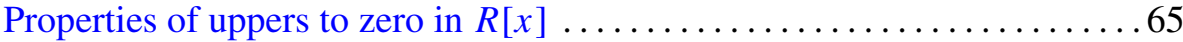

José M. Souto Menéndez, On a theorem due to Cassels ............. 81

Courtney Hughes Moen, Irreducibility of unitary principal series for

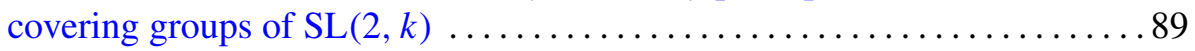

Frank M. Neubrander, Integrated semigroups and their applications to the

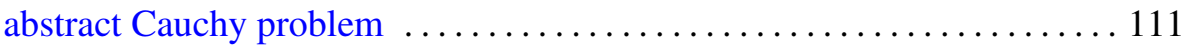

C. K. Qu and Roderick Sue-Chuen Wong, Szegő's conjecture on Lebesgue

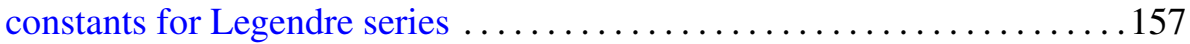

John Brendan Sullivan, The Euler character and cancellation theorems for

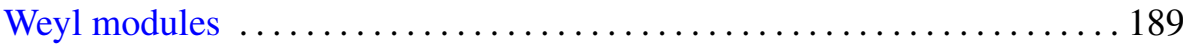

